\title{
Un testo sconosciuto di Jean Miélot: la traduzione dei "Vaticinia de summis pontificibus"
}

\section{Elisabetta Barale}

\section{(2) OpenEdition}

\section{Journals}

\section{Edizione digitale}

URL: http://journals.openedition.org/studifrancesi/285

DOI: 10.4000/studifrancesi.285

ISSN: 2421-5856

\section{Editore}

Rosenberg \& Sellier

\section{Edizione cartacea}

Data di pubblicazione: 1 aprile 2015

Paginazione: 63-74

ISSN: 0039-2944

\section{Notizia bibliografica digitale}

Elisabetta Barale, « Un testo sconosciuto di Jean Miélot: la traduzione dei "Vaticinia de summis pontificibus" », Studi Francesi [Online], 175 (LIX | I) | 2015, online dal 01 avril 2016, consultato il 18 septembre 2020. URL : http://journals.openedition.org/studifrancesi/285 ; DOI : https://doi.org/ 10.4000/studifrancesi.285

\section{(c) (i) (9)}

Studi Francesi è distribuita con Licenza Creative Commons Attribuzione - Non commerciale - Non opere derivate 4.0 Internazionale. 


\title{
Un testo sconosciuto di Jean Miélot: la traduzione dei "Vaticinia de summis pontificibus"
}

\begin{abstract}
This study is an initial attempt to investigate an unknown text by Jean Miélot: a Middle French translation of the Vaticinia de summis pontificibus transmitted by the manuscript Giessen, Universitätsbibliothek, Hs 633a. This series of illustrated Pope prophecies was attributed in 1862 to "Jo. Imelot" by Johan Valentin Adrian, who transliterated in this way the name of the writer and caused the volume to be forgotten for a long time.

In the first part of the article, results of an early codicological analysis are shared; then, the research provides an overview of the story of the Vaticinia Latin version, which encountered a remarkable success between the XV and the XVI centuries. A detailed central section aims to describe Miélot's peculiar layout and points out his interest both in vernacular and in the material aspect of the book. Finally, the study suggests some philological hypotheses: the use of more than one source as well as the adoption of a model possibly coming from Italy is discussed. In conclusion, the results of this study highlight the importance of the Giessen manuscript and support further researches. Being actually one of the first Vaticinia translations in vernacular, Miélot's text also improves our knowledge of his literary production and allows refining our notion of his working methods.
\end{abstract}

Gli studi pionieristici di Paul Perdrizet e di Robert Bossuat ${ }^{1}$ hanno guidato ricercatori provenienti da settori disciplinari diversi nella riscoperta di una figura di spicco nel panorama letterario borgognone del Quattrocento: Jean Miélot. Canonico della Collegiata di Saint-Pierre a Lille dal 1453, Miélot era stato nominato quattro anni prima «secrétaire aux honneurs» da Filippo il Buono e incaricato di «escripre et translater de latin en françois pluseurs livres et y faire pluseurs histoires» ${ }^{2}$ per la biblioteca ducale. L'esame della sua ampia e variegata produzione ${ }^{3}$ rivela che Miélot

(1) P. PERDRIZET, Jean Miélot, l'un des traducteurs de Philippe le Bon, «Revue d'histoire littéraire de la France», 14, 1907, pp. 472-482; R. BOsSUAT, Jean Miélot traducteur de Cicéron, «Bibliothèque de l'École des Chartes», 99, 1938, pp. 82-123.

(2) J. Paviot, Mention de livres, d'auteurs, de copistes, d'enlumineurs, de miniaturistes ("bistorieurs") et de libraires dans les comptes généraux du duc de Bourgogne Philippe le Bon (1419-1467), in Miscellanea in memoriam Pierre Cockshaw (19382008), II («Aspects de la vie culturelle dans les Pays-Bas Méridionaux»), Bruxelles, Archives et bibliothèques de Belgique, 2000, documento 153, p. 442 .

(3) L'opera di Miélot comprende la traduzione, la trascrizione, l'adattamento, la compilazione e la trasposizione in prosa di testi religiosi, morali e storici. A titolo esemplificativo, tra le traduzioni dal latino, ricordiamo il Miroir de la salvation bumaine (cfr. J. Lutz, P. Perdrizet, "Speculum bumanae salvationis". Texte critique. Traduction inédite. Les sources et l'influence iconographique principalement sur l'art alsacien du XIV' siècle, Mulhouse, Meininger, 19071909), l'Epistre que Tulle jadis envoia a son frere Quintus (cfr. S. LeFĖvRE, Jean Miélot, traducteur de la première "Lettre" de Cicéron à son frère Quintus, in La traduction vers le moyen français, Actes du II ${ }^{\mathrm{e}}$ Colloque de l'AIEMF [Poitiers, 27-29 aprile 2006], a cura di C. Galderisi e C. Pignatelli, Turnhout, Brepols, 2007, pp. 126-127), la Controverse de noblesse con il Desbat d'bonneur (cfr. A. VAnDERJAGT, Qui sa vertu anoblist: the concepts of "noblesse" and "chose publique" in Burgundian political thought including Fifteenth-century French translations of Giovanni Aurispa, Buonaccorso da Montemagno and Diego de Valera, Meppel, Krips Repro, 1981; A. Schoysman, Jean Miélot traducteur du "Débat de la vraie noblesse" de Buonaccorso da Pistoia, in La traduction vers le moyen français cit., pp. 323-336). Tra gli adattamenti, si può citare quello dell'Epistre Othea, trasmesso dai manoscritti Aylesbury, Waddesdon Manor, 8 e Bruxelles, kbr, 9392 (cfr. 
s'interessò a tutti gli aspetti della creazione del libro, dalla scelta delle fonti alle tecniche di compilazione, dall'impaginazione del testo alla coordinazione di elaborati progetti iconografici. La complessità di queste funzioni e l'assiduità con la quale il «prestre indigne» ${ }^{4}$ si prestò ad assolverle consentono di studiare le sue opere da molteplici punti di vista ${ }^{5}$, con la consapevolezza che l'analisi del singolo testo non può prescindere dall'aspetto materiale dei codici che lo trasmettono.

Questa nota metodologica è utile premessa allo studio di un'opera di Miélot finora sconosciuta: la traduzione in francese dei Vaticinia de summis pontificibus, una serie di trenta profezie papali illustrate, che nella versione latina ottenne un enorme successo nei manoscritti e nei libri a stampa tra XV e XVI secolo.

L'opera di Miélot è trasmessa dal ms. Giessen, Universitätsbibliothek, Hs 633a. Si tratta di un volume cartaceo di dimensioni abbastanza grandi $(26 \times 39 \mathrm{~cm})$, composto da 30 fogli. Le carte presentano sul recto, in alto a destra, una doppia numerazione moderna: la più antica, a penna, non corrisponde a quella più recente, a matita; la differenza tra i due sistemi induce a credere che, quando la numerazione più antica fu realizzata, le carte del manoscritto fossero slegate e in disordine. Il testo è vergato in inchiostro bruno in una grafia gotica bastarda corsiva, inclinata a destra. Il codice è caratterizzato da un ricco programma iconografico, che non è però stato ultimato: si contano ventinove disegni che occupano i tre quarti della facciata (ventidue righe di scrittura), ma il ventiduesimo è in realtà solo un abbozzo (c. 23r); inoltre, lo specchio di scrittura prevedeva spazi di varie dimensioni per l'inserimento di letterine, che non furono mai realizzate ${ }^{6}$.

Registrato per la prima volta da Johan Valentin Adrian (1793-1864) nell'Additamenta ad catalogum codicum manuscriptorum Bibliothecae Academicae Gissensis (1862), il testo del manoscritto era attribuito a "Jo. Imelot"; lo studioso tedesco trascrisse in questo modo l'«explicit»: «Fine la papaliste de l'abbé Joachm [sic] translaté de latin en françois par Jo. Imelot prestre chanoine de Lille... l'an de grace mil cccc $1 \mathrm{x} \gg^{7}$. Solo nel 1976, il nuovo catalogo attribuì correttamente il testo del codice a Jean Miélot ${ }^{8}$; tuttavia, questo inventario, redatto in forma dattiloscritta, ebbe diffusione limitata.

Delle due grafie, "Imelot" /"Mielot”, la seconda è l'unica autorizzata dall'autore stesso. In primo luogo, sembra possibile confermare che il vero nome dell'autore

G. Mombello, Per un'edizione critica dell'"Epistre Othea" di Christine de Pizan, «Studi Francesi», n. 24, 1964, pp. 401-417; A. SCHOYSMAN, Les deux manuscrits du remaniement de l'Epitre Othéa" de Christine de Pizan par Jean Miélot, «Le Moyen Français», 51-53, 2002-2003, pp. 505-528). Tra le compilazioni, le due serie dei Miracles de Nostre Dame comprendono anche le trasposizioni in prosa di quarantacinque miracoli originariamente in versi (cfr. L. Delisle, Les miracles de Notre-Dame. Rédaction en prose de Jean Miélot, «Bulletin historique et philologique du comité des travaux historiques et scientifiques», 1886, pp. 32-45).

(4) E in questo modo che Miélot si presenta in molte opere dedicate a Filippo il Buono e a Carlo il Temerario. L'appellativo si legge anche nella traduzione dei Vaticinia de summis pontificibus alla c. $30 \mathrm{v}$.

(5) Il fascicolo 67, 2011 della rivista «Le Moyen Français», che riunisce gli Atti di un Convegno su Miélot tenutosi nel 2009 all'Università di Siena (Te- sti, manoscritti e miniature: l'atelier di Jean Miélot), illustra i risultati che si possono ottenere in una prospettiva critica interdisciplinare.

(6) Una completa descrizione materiale del codice verrà fornita in sede d'edizione, quando potrà essere affrontata anche la questione dell'autografia/ autorialità del manoscritto, sulla base delle linee guida proposte da O. Delsaux, T. VAN Hemelryck, Les manuscrits autographes en français au Moyen Age. Guide de recherches. Avec trois articles de Gilbert Ouy, Turnhout, Brepols, 2014 («Texte, Codex \& Contexte», 15).

(7) J.V. ADRIAN, Additamenta ad catalogum codicum manuscriptorum Bibliothecae Academicae Gissensis, Giessen, 1862, p. 11.

(8) Katalog der Nachträge zum Gießener Handschriftenkatalog von Adrian (1840), 1840-1952, und der Anmerkungen zu den Handschriften 1840-1975, ora digitalizzato e disponibile sul sito della Biblioteca universitaria di Giessen. 
è "Mielot" e non "Imelot". Il codice Paris, BnF, fr. 17001, manoscritto autografo e personale di Miélot, contiene almeno tre riferimenti inequivocabili al suo nome. Percorrendo i labirinti disegnati sulle cc. 2v e 27r, si leggono, infatti, una dopo l'altra, le lettere che formano il nome "Mielot"; inoltre, gli studi di Pascal Schandel" hanno permesso di interpretare il rebus alla c. 5r, che rivela così il nome "Mielot" nascosto dietro ad una nota musicale (il "mi"), una sagoma a forma di "e" ed un recipiente che ha la capacità di un "lot". Nonostante queste prove che appaiono incontestabili, esiste un manoscritto che contiene una versione abbreviata e illustrata della Vie de saint Foursy redatta da Miélot ${ }^{10}$, nel quale il nome dell'autore appare tre volte: in tutte le occasioni, il primo tratto grafico sembra sormontato da una tilde, invitando a leggere il nome dell'autore come "Immelot". Una ricerca condotta sull'attestazione di questa grafia nei documenti storiografici ha rivelato che questa forma, riferita a Jean Miélot, si legge nella raccolta Scriptores Insulenses, conservata nel manoscritto settecentesco Lille, BM, $381^{11}$. Evidentemente, non tutti gli eruditi dei secoli passati hanno interpretato i grafemi corrispondenti al nome di Miélot nello stesso modo: di fronte a quattro trattini verticali, le due letture, "Imelot" e "Mielot", sono entrambe possibili"12.

Miélot cita i Vaticinia de summis pontificibus con il titolo di «Papaliste» e li attribuisce all'abate cosentino Gioacchino da Fiore (c. 1135-1202). In epoca tardo-medievale, i Vaticinia erano chiamati dai chierici "papalarius" o "paparium"13 e venivano registrati negli inventari in lingua volgare sotto il nome di «Libro Papalista» o «Papalisto» ${ }^{14}$; inoltre, si credeva che le profezie fossero il risultato delle rivelazioni ricevute da Gioacchino da Fiore sul fondo di un calice che un angelo gli aveva consegnato ${ }^{15}$. A questa leggenda, inventata per porre l'opera sotto l'autorità di un personaggio illustre, si deve in parte la popolarità del testo, le cui origini sono però più complesse.

Innanzi tutto va precisato che i Vaticinia sono un adattamento quattrocentesco di due testi, composti in momenti diversi e designati con i loro «incipit»: Genus nequam e Ascende calve ${ }^{16}$.

All'inizio del Novecento, le ricerche di Herbert Grundmann ${ }^{17}$ hanno dato avvio

(9) P. Schandel, "A l'euvre congnoist on l'ouvrier". Labyrinthes, jeux d'esprits et rébus chez Jean Miélot, in M. Hoffmann, C. ZöHL, Quand la peinture était dans les livres. Mélanges en l'bonneur de François Avril, Turnhout, Brepols, 2007, pp. 295-302.

(10) Questo volume, di piccole dimensioni e composto da soli quindici fogli, ha fatto parte della collezione di Bernard Blondeel ad Anversa fino al 1986 e si trova attualmente in mani private. Lo studio completo della Vie de saint Foursy, opera agiografica in prosa la cui redazione interessò Miélot dal 1462 al 1469 , è confluito nella mia tesi di dottorato: Édition critique de "La genealogie, la vie, les miracles et les merites de saint Fursy" de Jean Miélot (ms. Wien, Österreichische Nationalbibliothek, Series Nova 2731), sotto la direzione di P. Cifarelli e T. VAN HeMELRYCK, Università degli Studi di Torino/ Université Catholique de Louvain, maggio 2014. La tesi è in corso di revisione in vista della pubblicazione.

(11) «Joannes Immelot, canonicus insignis collegiatae S. Petri Insulis, anno 1458, ad instantiam potentissimi Burgundionum ducto, Philippi boni, vertit e latino in gallicum sermonem vitam $S$. Adriani martyris atque miracula, quae Deus per sui martyris merita patrarat Gerardimonti: exstant manuscripta perantiqua cum figuris vitam et ejus martyrium apud D. Eubertum Guilielmum Scherer de Cherbourg, equitem, toparcham de Tourmignies, de Leprez, etc.», in M. LE GLAY, Catalogue descriptif des manuscrits de la bibliothèque de Lille, Lille, Vanackere, 1848, p. XXVII.

(12) Bertrand Schnerb considera "Imelot" come un alias di Miélot. B. SCHNERB, La piété et les dévotions de Philippe le Bon, duc de Bourgogne (1419-1467), «Comptes-rendus des séances de l'Académie des Inscriptions et Belles-Lettres», 149, 2005, pp. 1319-1344, in particolare p. 1331, nota 71; A.M. Legaré, B. SCHNERB, Livres et lectures de femmes en Europe entre Moyen Age et Renaissance, Turnhout, Brepols, 2007, pp. 235 e 346.

(13) H. Millet, "Il libro delle immagini dei Papi". Storia di un testo profetico medievale, Roma, Viella, 2002 , p. 125 . Il volume è stato edito anche in lingua francese: ID., Successeurs du pape aux ours. Histoire d'un livre propbétique médiéval illustré ("Vaticinia de summis pontificibus"), Turnhout, Brepols, 2004.

(14) D. RINI, I "Vaticinia de summis pontificibus" e la copia del "Genus nequam" dell'Art Institute of Chicago, Scuola di specializzazione in Storia dell'Arte, Università di Pisa, a.a. 2009-2010, p. 36.

(15) H. Millet, "Il libro delle immagini dei Papi". Storia di un testo profetico medievale cit., p. 217.

(16) Ivi, pp. 79-98.

(17) H. Grundmann, Die Papstprophetien des 
ad un appassionante filone di studi che, grazie ai contributi di numerosi esperti ${ }^{18}$, ha fatto luce sulla genesi di Genus nequam. In particolar modo, è stato possibile scoprire che, non oltre la primavera del 1287, in ambiente angioino si realizzò probabilmente il primo adattamento in chiave ecclesiastica degli Oracoli di Leone, un'opera politica greca illustrata, giunta in Occidente intorno al 1274 o poco più tardi ${ }^{19}$. Sei criptiche strofe sui sovrani orientali furono tradotte e reinterpretate: venne così redatta un'invettiva contro i cardinali della famiglia Orsini, accusati di corruzione e nepotismo (Oracoli dei cardinali). Tra il 1280 e il $1305^{20}$ maturò poi l'idea di riprendere integralmente gli Oracoli di Leone e di attribuirli ai papi che avevano raccolto l'eredità di Niccolò III: nacque così la serie Genus nequam ${ }^{21}$. Il libello, composto da un numero di profezie variabile da quattordici a sedici, riprendeva essenzialmente i testi e le immagini del modello greco, limitandosi ad amplificare i titoli e a inserire gli emblemi pontificali nei disegni. In questo modo, gli imperatori bizantini diventavano dei papi e la prospettiva escatologica, da essi originariamente veicolata, poteva essere usata per celebrare la positiva venuta messianica dell'ultimo papa.

Nella prima metà del Trecento, probabilmente intorno agli anni Venti del seco$1 \mathrm{o}^{22}$, comparve l'Ascende Calve, la seconda serie di profezie papali². Ancora oggi si ritiene valida l'ipotesi espressa da $\mathrm{H}$. Grundmann, secondo la quale il testo sarebbe stato prodotto da un nucleo di francescani spirituali a chiari fini propagandistici. Composto da quindici profezie, questo gruppo riproponeva la sequenza del Genus nequam, ma si caratterizzava per la presenza di immagini rinnovate e di testi, intessuti di citazioni bibliche, che ribaltavano il messaggio dell'antecedente: pur partendo da Niccolò III, la successione dei papi terminava, infatti, in un senso profondamente pessimistico e anticlericale, con la bestia dell'Anticristo ${ }^{24}$.

Mittelalters, «Archiv für Kulturgeschichte», 19, 1929 , pp. 77-138. L'articolo è stato ristampato in: ID., Ausgewählte Aufsätze, vol. II: Joachim von Fiore, Stuttgart, Hiersemann, 1977, pp. 1-57.

(18) Tra i principali studi è possibile menzionare: A. Daneu-lattanzi, I "Vaticinia Pontificum" ed un codice monrealese del sec. XIII-XIV, «Atti della Reale Accademia di scienze, lettere e arti di Palermo», Ser. 4,3, 2/4, 1944, pp. 757-792; M. ReEves, The Influence of Prophecy in the Later Middle Ages. A study in Joachimism, Oxford, Clarendon Press, 1969; E.R. LeRnER, Weissagungen über die Päpste: Vat. Ross. 374 (Eine Einfübrung), Stuttgart, Belser Verlag, 1985; E.R. LERNER, On the Origins of the Earliest Latin Pope Prophecies: A Reconsideration, in Falschungen im Mittelalter, Internationaler Kongress der Monumenta Germaniae Historica [Monaco, 16-19 settembre 1986], Hannover, Hahnsche Buchhandlung, 1988-90, pp. 611-635; A. REHBERG, Der "Kardinalsorakel"-Kommentar in der "Colonna"-Handschrift Vat. lat. 3819 und die Entstehungsumstände der Papstvatizinien, «Florensia», 5, 1991, pp. 45-112; M. ReEves, The "Vaticinia de summis pontificibus": a Question of Authority, in Intellectual Life in the Middle Ages: Essays Presented to Margaret Gibson, London, Rio Grande, Ohio, Hambledon, 1992, pp. 145-156; H. Millet, D. Rigaux, Aux origines du succès des "Vaticinia de summis pontificibus", in Fin du monde et signes des temps, visionnaires et prophètes en France méridionale (fin du XIII-début XV siècle), Toulouse, Privat, 1992 («Cahiers de Fanjeux», 27), pp. 129-156; E.R. LERNER, Recent Work on the Origins of the "Genus
Nequam” Prophecies, «Florensia», 7, 1993, pp. 141 157; F. TRONCARELlI, "Vaticinia pontificum". Dal Codice 25. F 16 [recte 17] della Biblioteca Comunale di Monreale, Bari, Mario Adda, 2007; G.L. PotestÀ, L'uomo con la falce e la rosa. Dagli "Oracula Leonis" ai "Vaticinia pontificum" della Biblioteca Estense, in Profezie illustrate gioachimite alla corte degli estensi, Modena, Panini, 2010, pp. 131-179.

(19) G.L. Potestà, L'ultimo messia, Bologna, Il Mulino, pp. 159-160.

(20) E.R. LERNER, Recent Work on the Origins of the "Genus Nequam" Prophecies cit., pp. 155-156.

(21) Per un'edizione critica di Genus nequam si può far riferimento a M. Fleming, The Late Medieval Pope Prophecies. The "Genus nequam" Group, Tempe, Arizona Center for Medieval and Renaissance Studies, 1999.

(22) G.L. Potestà, L'ultimo messia cit., p. 178.

(23) Edizione critica di: O. SCHWARTZ, e.R. LERNER, Illuminated Propaganda: The Origins of the "Ascende calve" Pope Prophecies, «Journal of Medieval History», 20/2, 1994, pp. 157-191; ristampa in italiano: ID., Propaganda miniata: le origini delle profezie papali "Ascende Calve", Milano, Biblioteca Francescana, 1994. Inoltre, si vedano gli studi di: R. Blumenfeld-Kosinski, Poets, Saints and Visionaries of the Great Schism 1378-1417, Pennsylvania, Pennsylvania State University, 2006, in particolare pp. 165-200 e di H. Millet, D. Rigaux, "Ascende Calve”. Quand l'bistorien joue au prophète, «Studi Medievali», 33, 1992, pp. 695-719.

(24) G.L. POTESTÀ, L'ultimo messia cit., p. 178. 
Fu proprio la possibilità di attribuire al testo delle profezie nuovi significati a favorire la creazione dei Vaticinia de summis pontificibus. Nei difficili anni del Grande Scisma d'Occidente (1378-1417), e in particolar modo all'epoca del concilio di Costanza (1414-1417) o nel periodo immediatamente precedente ${ }^{25}$, le due serie profetiche furono riunite, invertendone l'ordine rispetto alle relative date di composizione e orientando in questo modo il senso dell'opera in prospettiva filo-papale ${ }^{26}$; in altre parole, i promotori della nuova raccolta ribadivano la loro fedeltà alla Santa Sede e si auguravano l'avvento di un pontefice in grado di riportare unità in seno alla Chiesa. I Vaticinia si componevano allora di trenta profezie, in genere conformi a quelle trasmesse dalle serie originali, salvo qualche piccolo intervento iconografico. L'opera aveva come obiettivo di invitare i lettori a cogliere sottili allusioni storiche sui pontefici, mediante un gioco di confronti: se Urbano VI, il fautore del Grande Scisma, finiva per corrispondere al dragone dell'Anticristo, come si sarebbe dovuto interpretare il ruolo di Giovanni XXIII, la cui immagine assomigliava a quella del dimissionario Celestino V? È probabile supporre che i redattori del libello fossero vicini agli ambienti della curia e che avessero lo scopo di spingere Giovanni XXIII all'abdicazione ${ }^{27}$. In effetti, quando quest'ultimo fu destituito, l'obiettivo primario della diffusione dei Vaticinia si esaurì, ma ne emerse uno nuovo: i numerosi manoscritti realizzati sotto il pontificato di Martino V, Eugenio IV e Niccolò V lasciano così trasparire grande riguardo nei confronti dell'autorità ecclesiastica e rinnovata fiducia in un papato in piena rinascita ${ }^{28}$. La storia dei Vaticinia, documentata dai trenta codici conservati ${ }^{29}$, è quindi quella di un best-seller. Quanto alla loro diffusione vernacolare, nonostante la scarsità degli studi sulla ricezione dell'opera nelle lingue moderne, sembra possibile affermare che, tra le più antiche traduzioni in volgare ${ }^{30}, \mathrm{ci}$ fu quella in italiano ad opera del domenicano Leandro Alberti, stampata a Bologna da Girolamo Benedetti nel 1515, in un'edizione identica dal punto di vista editoriale a quella latina apparsa in contemporanea ${ }^{31}$. Appare quindi ancora più rilevante che il manoscritto Giessen, Universitätsbibliothek, Hs 633a trasmetta una traduzione in francese anteriore di oltre cinquant'anni, datata 1460. Tenendo conto che in Francia i Vaticinia furono inizialmente accolti con freddezza, perché il paese si batté a lungo per il trionfo dell'obbedienza avignonese, e che solo nel XVII secolo l'opuscolo riuscì a beneficiare di maggiore considerazione ${ }^{32}$, la traduzione di Miélot desta grande inte-

(25) H. Millet, "Il libro delle immagini dei Papi". Storia di un testo profetico medievale cit., pp. 111136 , in particolare p. 112.

(26) E.R. LERNER, Weissagungen über die Päpste: Vat. Ross. 374 (Eine Einfübrung) cit., p. 60; P. GuERRINI, L'Anticristo "Bestia terribilis" nelle profezie figurate del Quattrocento e del Cinquecento, in Il profetismo gioachimita tra Quattrocento e Cinquecento, a cura di G.L. Potestà, Genova, Marietti, 1991 , pp. $87-96$, in particolare p. 88 .

(27) H. Millet, "Il libro delle immagini dei Papi". Storia di un testo profetico medievale cit., p. 134.

(28) Ivi, p. 183.

(29) G.L. POTESTÀ, L'uomo con la falce e la rosa. Dagli "Oracula Leonis" ai "Vaticinia pontificum" della Biblioteca Estense cit., p. 131. Per un elenco completo dei manoscritti che trasmettono le serie profetiche, consultare H. Millet, "Il libro delle immagini dei Papi". Storia di un testo profetico medievale cit., pp. 260-262.

(30) Il prof. Robert Lerner, che ringrazio senti- tamente per le informazioni che mi ha fornito, conferma che ad oggi non si conoscono volgarizzamenti anteriori al XVI secolo.

(31) Le edizioni gemelle avevano questi titoli: Iachimi abbatis Vaticinia circa apostolicos viros et Eccle(siam) R(omanam) e Prophetia dello Abbate Ioachino circa li pontifici et R(omana) C(biesa). Cfr. R. RusCONI, "Ex quodam antiquissimo libello". La tradizione manoscritta delle profezie nell'Italia tardomedievale: dalle collezioni profetiche alle prime edizioni a stampa, in The use and abuse of eschatology in the Middle Ages, Leuven, Leuven University Press, 1988, pp. 441-472, in particolare p. 461 (ripubblicato in R. Rusconi, Profezia e profeti alla fine del Medioevo, Roma, Viella, 1999, pp. 161-186). La più famosa traduzione in italiano dei Vaticinia è tuttavia quella di Pasqualino Regiselmo, pubblicata a Venezia nel 1589 , insieme al testo latino. Cfr. H. Millet, "Il libro delle immagini dei Papi". Storia di un testo profetico medievale cit., p. 244.

(32) Ivi, pp. 124-126. 
resse: potrebbe trattarsi infatti di uno dei primi tentativi di trasposizione dell'oscura materia profetica in lingua francese ${ }^{33}$.

L'«explicit» rivela che Miélot si avvicinò al testo dei Vaticinia con prudente deferenza. Definendo le profezie come «scabreuses, incorrectes et moult obscures», l'autore si propone di presentarle «sans y riens adjouster du sien qui detraye a la matiere» $[\mathrm{c} .30 \mathrm{v}]$. Il consueto atteggiamento di rispetto nei confronti dell'originale latino, percepito come un'auctoritas da seguire scrupolosamente nel timore di tradire il significato delle profezie ${ }^{34}$, si declina tuttavia nell'opera di Miélot in maniera originale. I Vaticinia sono tradizionalmente composti da tre elementi: l'immagine, il titolo $^{35}$ e il testo profetico; ad essi si aggiunge talvolta l'attribuzione degli oracoli ai papi, dato non sempre presente nei manoscritti latini. Se da un lato Miélot scelse di mantenere questa struttura, dall'altro decise di adottare soluzioni d'impaginazione autonome e moderne $e^{36}$. La disposizione di queste componenti sulla pagina merita dunque di essere osservata nel dettaglio. Ogni profezia occupa due facciate ad iniziare sul verso del foglio; in questo modo, a ciascun papa viene riservato uno spazio visualmente uniforme e al lettore si consente di impegnarsi nella decifrazione del testo, senza interrompersi per voltare pagina. Sul verso di ogni foglio si leggono dunque: il nome di battesimo del pontefice in alto, al centro; la dicitura «Prophecie» seguita dal titolo in latino e in francese; la traduzione francese del testo profetico; il testo profetico latino ${ }^{37}$. Sul recto, invece, si trovano: il nome assunto dal pontefice in alto, al centro; l'immagine; la dicitura «Extrait de la Cronique martinienne», seguita da una breve biografia del papa. È molto probabile che Miélot avesse colto nella specificità strutturale dei Vaticinia la possibilità di sperimentare le tecniche di "mise en page" che gli erano care: gli studi effettuati sulle sue opere, in particolare quelli sull'adattamento dell'Epistre Othea ${ }^{38}$ e sui VII Sacremens de l'Eglise ${ }^{39}$, hanno infatti evidenziato come Miélot s'impegnasse costantemente nel tentativo di ottenere impaginazioni molto regolari quanto al numero di righe destinate al testo e alla posizione delle immagini, fattori chiamati a svolgere un ruolo fondamentale nell'organizzazione della pagina. La lettura completa del «Papaliste» conferma la preoccupazione estetica di un autore/copista in cerca di efficaci soluzioni formali per i suoi volumi. Di fatto, è possibile notare che, nel caso di dodici oracoli ${ }^{40}$, il testo latino non è stato copiato per

(33) Per descrivere la sua opera, Miélot usa l'espressione «translaté de latin en françois» [c. 30v].

34) D. RINI, I "Vaticinia de summis pontificibus" e la copia del "Genus nequam" dell'Art Institute of Chicago cit., p. 43.

(35) In accordo con E.R. Lerner (ID., Recent Work on the Origins of the "Genus Nequam" Prophecies cit., pp. 150-151, n. 20), ho scelto di adottare il termine "titolo" al posto di "rubrica" perché i titoli, sebbene fisicamente separati dal testo profetico, non sono propriamente "rubricati".

(36) Questo implicherebbe l'autografia/autorialità del manoscritto. Si rinvia pertanto alla nota 6 .

(37) L'unica eccezione a quest'ordine è costituita dalla quindicesima profezia, nella quale il nome di battesimo del pontefice è seguito dal testo profetico latino, dalla traduzione francese e, infine, dalla dicitura «Prophecie» con il titolo e la sua traduzione.

(38) R. BROWN-GRANT, Illumination as reception. Jean Miélot's reworking of the "Epitre Othéa", in The city of scholars. New approaches to Christine de Pizan, a cura di M. Zimmermann, D. De RentiIs, Berlin, De Gruyter, 1994, pp. 260-271; A. SCHOysman,
L'écriture mythographique de l'bistoire à la cour de Bourgogne. Les "Genealogie deorum gentilium" de Boccace exploitées par Miélot, remanieur de l'"Epitre Othea" de Christine de Pizan, in Ecritures de l'bistoire (XIV'-XVI siècle), Actes du Colloque du Centre Montaigne [Bordeaux, 19-21 settembre 2002], éd. D. Bohler, C. Magnien-Simonin, Genève, Droz, 2005, pp. 73-82; T. Van Hemelryck, C. Van HoOREBEECK, L'Epistre Othea" de Christine de Pizan par Jean Miélot, «Le Moyen Français», 67 cit., pp. 111128.

(39) M. Colombo Timelli, "Les VII Sacremens de l'Eglise". Édition critique, «Studi Francesi», n. 163, pp. 63-81; M. Colombo Timelli, "Les VII Sacremens de l'Eglise" de Jean Miélot: une ceuvre "originale"?, in Original et originalité, Colloque international du Groupe de recherche sur le moyen français et de l'AIEMF [Louvain-la-Neuve, 20-22 maggio 2010], Louvain-la-Neuve, Presses Universitaires de Louvain, 2012, pp. 45-52.

(40) Si tratta delle profezie 4, 6, 7, 15, 17, 18, 19 , $22,23,26,27,28$. 
intero sulla pagina di sinistra: questo avviene soprattutto in corrispondenza di testi profetici lunghi, la cui traduzione soprastante occupa molto spazio. E forse azzardato supporre che i testi latini siano stati inseriti per riempire degli spazi che sarebbero altrimenti rimasti vuoti, ma è evidente che il copista attribuiva importanza maggiore alla versione vernacolare, copiata per intero. Ed è sempre con un occhio di riguardo all'aspetto materiale del libro che venne probabilmente scelto di inserire, sotto le effigi dei pontefici, alcuni estratti di una delle continuazioni della Cronaca martiniana ${ }^{41}$. In effetti, le immagini dei papi occupano quasi tutta la pagina, ma lasciano libere otto righe, che vennero sapientemente riempite con aneddoti storici e letterari utili ad interpretare gli oracoli. Non è da escludere, infatti, che un'altra delle ragioni per cui Miélot s'interessò ai Vaticinia fosse proprio la loro enigmatica natura: riunendo gli indizi disseminati tra immagini e testi, Filippo il Buono, il raffinato mecenate al quale l'opera è dedicata, avrebbe potuto interpretare i "profetici" indovinelli, traendo piacere dallo sforzo intellettuale.

Per quanto riguarda il contenuto dell'opera, la tabella illustra le profezie secondo l'ordine in cui si presentano nel manoscritto di Giessen.

$\begin{array}{cll}\text { Profezie } & \text { Pontefice } & \text { Tratti salienti delle immagini } \\ 1 & \text { Niccolò III }(1277-1280) & \text { Papa che nutre due orsi } \\ 2 & \text { Martino IV }(1281-1285) & \text { Aquila trafitta dal giglio } \\ 3 & \text { Onorio IV }(1285-1287) & \text { Uomo con bastone } \\ 4 & \text { Niccolò IV }(1288-1292) & \text { Due dame con drago } \\ 5 & \text { Celestino V }(1294-1296) & \text { Volpe che cerca di strappare la tiara } \\ & & \text { al papa } \\ 6 & \text { Bonifacio VIII }(1294-1303) & \text { Gallo e piccolo ecclesiastico } \\ 7 & \text { Benedetto XI }(1303-1304) & \text { Serpente tentatore } \\ 8 & \text { Clemente V }(1305-1314) & \text { Cavaliere che abbandona la dama } \\ 9 & \text { Giovanni XXII }(1316-1334) & \text { Papa con spada in bocca e antipapa } \\ & & \text { con la mitra } \\ 10 & \text { Benedetto XII }(1334-1342) & \text { Colomba e sei stelle } \\ 11 & \text { Clemente VI }(1342-1352) & \text { Gambe del papa in un calderone } \\ 12 & \text { Innocenzo VI }(1352-1362) & \text { Chiavi, rasoio e lupo } \\ 13 & \text { Urbano V }(1362-1370) & \text { Pavone e angelo } \\ 14 & \text { Urbano VI }(1378-1389) & \text { Anticristo con serpente e scorpione } \\ 15 & \text { Bonifacio IX }(1389-1404) & \text { Papa con due orsi } \\ 16 & \text { Innocenzo VII }(1404-1406) & \text { Serpente che lotta con uccello } \\ 17 & \text { Gregorio XII }(1406-1415) & \text { Ragazzo, uccello e unicorno } \\ 18 & \text { Alessandro V }(1409-1410) & \text { Tre colonne con testa coronata, testa } \\ & \text { - eletto al concilio di Pisa } & \text { con tonsura e mezzaluna } \\ 19 & \text { Giovanni XXIII (1410-1419) } & \text { Falce, fiore, lettera B e gamba } \\ & \text { - eletto al concilio di Pisa } & \\ 20 & \text { Martino V (1417-1431) } & \text { Vacca } \\ 21 & \text { Eugenio IV }(1431-1447)^{42} & \text { Orsa }\end{array}$

(41) L’identificazione della continuazione della Cronaca martiniana che fu utilizzata come fonte richiede ulteriori ricerche. Per il momento, ci si può limitare a rilevare la presenza di indicazioni non corrette, come alcune date o il luogo di nasci-

ta di Bonifacio VIII (Avignone invece di Anagni), che potrebbero essere dovute tanto ad un errore presente nel modello quanto ad una cattiva lettura di Miélot o ancora ad una trascrizione sbagliata da parte del copista del manoscritto di Giessen. 
Città con guardie

(disegno incompiuto)

23

24

25

26

27

28

$29+30$

+ «autre»

prophecie
Città e mani tese

Volpe

Papa nudo

Quattro canidi posti di tergo

Papa incoronato dall'angelo

Tendaggio sostenuto da due angeli

Animale incoronato simile a un leone

e a un montone

Questa galleria dei papi manifesta tre evidenti particolarità. Innanzi tutto, l'ultimo pontefice a cui è stata chiaramente attribuita una profezia è Eugenio IV: in linea di principio, è possibile ritenere che l'ultimo papa menzionato è quello in carica al momento in cui il codice viene redatto. Nel caso del manoscritto di Giessen, datato 1460, tuttavia, le attribuzioni si fermano al 1455, ignorando ben due pontefici: Callisto III (Alfonso Borgia, 1455-1458) e Pio II (Enea Silvio Piccolomini, 1458-1464). L'ultima attribuzione a un papa la cui missione terrena si era conclusa da circa dieci anni potrebbe dunque dare informazioni sulla fonte usata da Miélot. Le profezie successive, prive di uno specifico riferimento, corrisponderebbero ai papi del futuro, suggerendo ai lettori la possibilità di continuare a cogliere le coincidenze della storia ${ }^{43}$.

La seconda caratteristica di questa successione di pontefici è che, sebbene il canonico piccardo avesse manifestato nel prologo il desiderio di esporre le profezie associate a trenta papi «passez et advenir» ${ }^{44}$, il manoscritto presenta solo le immagini di ventinove pontefici. Infatti, alla c. $14 \mathrm{v}$ s'incontrano il nome e la profezia di Pierre d'Estoillefort, verosimilmente quel Pierre Roger de Beaufort che divenne Gregorio XI (1370-1378), mentre alla c. 15r si leggono il nome di Urbano VI e la sua biografia. $\mathrm{Ne}$ consegue che è possibile supporre l'esistenza di una lacuna materiale, causata dalla perdita di un foglio su tallone. Il recto della carta mancante avrebbe potuto presentare l'immagine e la biografia di Gregorio XI; il verso avrebbe invece trasmesso il nome di battesimo e la profezia di Urbano VI.

Infine, è utile segnalare un terzo aspetto. Concordemente alla tradizione dei $\mathrm{Va}$ ticinia, il libello di Miélot si chiude presentando una fusione testuale e iconografica delle profezie 15 e 16 della serie Genus Nequam ${ }^{45}$. L'impaginazione mostra, tuttavia, un trattamento particolare dei testi latini: la c. 29v riporta due profezie (29 e 30) e aggiunge quella che Miélot chiamò l'«autre prophecie»; dietro a questa apparente complessità, si nasconde di fatto una riorganizzazione degli oracoli del Genus Nequam ${ }^{46}$, già presente con ogni probabilità nel modello usato dall'autore.

(42) Eugenio IV è l'ultimo papa il cui nome compare per esteso nel manoscritto e di cui è inserita una biografia. Del successore, Niccolò V (14471455), al secolo Tommaso Parentucelli, si legge solo parte del nome di battesimo: "Thomas" [c. 22r].

(43) D. RinI, I "Vaticinia de summis pontificibus" e la copia del "Genus nequam" dell'Art Institute of Chicago cit., p. 31.

(44) «Cy commence ung livre nommé le Papaliste, jadis fait et composé en vision par l'abbé Joachin, touchant les prophecies et advenues de XXX papes passez et ad venir» [c. 1r].

(45) H. Millet, "Il libro delle immagini dei Papi".
Storia di un testo profetico medievale cit., pp. 169 170.

(46) Il titolo della ventinovesima profezia corrisponde alla seconda parte del testo profetico del sedicesimo oracolo di Genus nequam, mentre il suo testo è quello del quindicesimo oracolo; la trentesima profezia, composta da un solo elemento, è il titolo del quindicesimo oracolo; l'«autre prophecie», anch'essa composta da un solo elemento, è la prima parte del testo profetico del sedicesimo oracolo. Le tre profezie vengono associate all'immagine del papa con la bestia coronata, metà leone, metà montone. 
L'individuazione delle fonti che Miélot ebbe modo di consultare non è priva d'interesse. I cataloghi pubblicati da Barrois non segnalano la presenza di manoscritti contenenti i Vaticinia de summis pontificibus nella biblioteca dei duchi di Borgogna ${ }^{47}$. Un primo esame del «Papaliste» consente tuttavia di formulare delle considerazioni che si fondano su argomenti iconografici e traduttologici, e quindi di aprire alcune piste di ricerca.

Hélène Millet e Dominique Rigaux hanno analizzato i codici del xv secolo che trasmettono i Vaticinia e li hanno suddivisi in tre famiglie: Arundel, Regina ed Este ${ }^{48}$. Il criterio principale alla base della classificazione è l'ordine in cui compaiono i vaticinia da 21 a 26. Il manoscritto di Giessen presenta le immagini corrispondenti a quelle profezie in questa sequenza: vacca; orsa; città con guardie; città e mani tese; volpe; papa nudo. L'ordine è il medesimo dei manoscritti del gruppo Regina. Il confronto dei disegni del codice di Giessen con quelli del gruppo Regina conferma questa relazione. Tra i numerosi punti di contatto, si possono ricordare alcuni motivi iconografici: l'antipapa che indossa la mitra nella profezia 9 (nelle versioni Arundel ed Este, l'antipapa indossa una tiara); la mezzaluna sulla colonna di destra nella profezia 18 (nelle versioni Arundel ed Este, c'è la lama di una falce rivolta verso la testa appoggiata sulla colonna centrale); le sembianze di leone e montone dell'animale coronato nell'ultima profezia (nelle versioni Arundel ed Este, l'animale è un cervide peloso). H. Millet ha sottolineato che le scelte iconografiche adottate dai redattori del gruppo Regina si caratterizzano per una sorta di «ritorno alle origini». In altre parole, mentre i compilatori del ramo Arundel-Este manifestarono maggiore fantasia creativa, quelli del ramo Regina preferirono riprendere le immagini degli archetipi ${ }^{49}$, ovvero di Genus nequam e di Ascende calve ${ }^{50}$. Altri studi volti a identificare l'area di diffusione dei codici hanno dimostrato che il nord e il centro Italia costituirono i fulcri della produzione tardiva ${ }^{51}$. In conclusione, tenendo presente che il manoscritto di Giessen

(47) Secondo H. Wijsman, che non dà peraltro ulteriori precisazioni, né rinvii agli inventari editi da Barrois, un manoscritto latino, realizzato in Germania intorno al 1415 ed intitolato Vaticinia sive prophetiae et imagines summorum pontificium, avrebbe fatto parte della biblioteca dei duchi di Borgogna (cf. H. Wijsman, Bibliothèques princières entre Moyen Age et Humanisme. A propos des livres de Philippe le Bon et de Matthias Corvin et de l'interprétation du XV siècle, in Matthias Corvin, les bibliothèques princières et la genèse de l'état moderne, a cura di J.-F. MAILLARD, I. MonoK, D. NebBiaI, Budapest, Országos Széchényi Könyvtár, 2009, pp. 121-134, in particolare p. 131). In ogni caso, da un punto di vista iconografico, il manoscritto in questione, attualmente Paris, BnF, lat. 10834, non può essere messo in relazione con il manoscritto di Giessen perché appartiene ad un'altra famiglia testuale (Arundel). Cf. L. Von WILCKENS, Die Prophetien über die Päpste in deutschen Handschriften. Zu Illustrationen aus der Pariser Handschrift Lat. 10834 und aus anderen Manuskripten der ersten Hälfte des 15. Jahrbunderts, «Wiener Jahrbuch für Kunstgeschichte», 28, 1975, pp. 171-180. Infine, ritengo opportuno segnalare che i cataloghi pubblicati da Barrois registrano tre manoscritti consacrati alla storia dei pontefici: due volumi intitolati C'est le livre historié des Papes, Emprereurs et Cardinaulx (numeri 968 e 1951) e un volume dal titolo Histoire de pluseurs Papes (num. 2076); la presenza di queste opere nella biblioteca ducale sarà oggetto d'indagine più approfondita in sede di edizione. Cf. J.B.J. BARROIs, Bibliothèque protypographique, ou Librairies des fils du roi Jean, Charles V, Jean de Berri, Philippe de Bourgogne et les siens, Paris, Treuttel et Würtz, 1830, pp. 153,278 e 295 .

(48) Il nome attribuito a ciascuna delle famiglie fa riferimento alla segnatura dei testimoni antichi più rappresentativi. H. Millet, "Il libro delle immagini dei Papi". Storia di un testo profetico medievale cit., pp. 64-67.

(49) L'unica innovazione imputabile a questi redattori è l'introduzione della mezzaluna sulla colonna di destra nella profezia 18 , stemma di famiglia di Benedetto XIII. Cfr. H. Millet, "Il libro delle immagini dei Papi". Storia di un testo profetico medievale cit., pp. 145-146.

(50) Queste soluzioni iconografiche arcaiche, abbinate a un notevole rispetto nei confronti dell'autorità papale, hanno condotto la studiosa francese a situare lorigine della famiglia Regina nel periodo successivo alla deposizione di Giovanni XXIII: d'altronde, la maggior parte dei manoscritti del gruppo venne redatta alla fine del Grande Scisma, sotto il pontificato di Eugenio IV (1431-1477). Le famiglie Arundel-Este testimonierebbero, per contro, della fase più antica della diffusione dei Vaticinia. H. Millet sottolinea, in particolare, che nessun testimone della famiglia Arundel venne realizzato dopo il pontificato di Giovanni XXIII. Ivi, pp. 142 e 183-186.

(51) L'origine tedesca è invece dominante nella prima fase di diffusione dei Vaticina: tre dei cinque esemplari più antichi furono realizzati nella Germania meridionale. Ivi, p. 128. 
s'inserisce nella tradizione della famiglia Regina, è verosimile supporre che Miélot abbia potuto usare come modello un testimone di questo gruppo, redatto probabilmente in Italia in anni recenti.

Già R. Bossuat e G. Mombello avevano notato un grande interesse da parte del canonico di Lille per l'attività letteraria degli umanisti d'oltralpe ${ }^{52}$. In questo caso, la possibile origine italiana del modello parrebbe supportata da un significativo dato di natura testuale. Per esaminarlo, è opportuno leggere per esteso il testo profetico del ventunesimo oracolo, in francese e in latino [c. $21 \mathrm{v}$ ]:

[L'] autre ourse seconde sera paissant ses cayaulx, c'est a dire ses petis ourseaulx, en tous lieux et places fors que en l'umbre. Touteffois la nature des temps de sa nativité est escripte abortive, car en son dernier eage elle sera navree d'un audrag ulcereux, c'est assavoir de pape Felix, esleu au concile de Basle. Certes les tribulations subsolaires manifesteront a l'un et a l'autre des corbeaulx la division de toute puissance en la reduction des Grecz au concile de Ferraire etc. ${ }^{53}$

[A]lia ursa secunda catulos pascens et in omnibus illa preterquam in umbra. Tamen scripta natura temporum nativitatis abortiva. In ultima enim scribitur ultime subsolares aut utrumque cor hominis manifestans divisiones totius produne [sic] etc.

La traduzione di Miélot presenta alcune differenze rispetto al testo profetico del vaticinium. Dopo una glossa lessicografica («cayaulx» è la forma piccarda di «cael», che significa "piccolo di un animale", ma assume in questo caso il senso specifico di "cuccioli dell'orsa"), l'aggiunta di «car en son dernier eage elle sera navree d'un audrag ulcereux $\gg{ }^{54}$ chiarisce in che senso la natura dei tempi è abortiva. I testi medici di fine Quattrocento fanno riferimento all'«audrag» come ad una piccola pustola purulenta $^{55}$; in questa profezia, esso è addirittura «ulcereux» e ha dunque la capacità di ferire profondamente e di espandersi ${ }^{56}$. Un'altra glossa, «c'est assavoir de pape Felix esleu au concile de Basle», esplicita la metafora: la pustola ulcerosa è il duca di Savoia Amedeo VIII (1383-1451), eletto antipapa con il nome di Felice V al concilio di Basilea nel 1439. L'aggiunta, «en la reduction des Grecz au concile de Ferraire», permette poi di contestualizzare l'ingiuria: durante il Piccolo Scisma d'Occidente (1439-1449), nelle aree dell'Italia centro-settentrionale, vicino alle città di Ferrara e Firenze, in cui si riunirono le assemblee destinate ad opporsi ai prelati di Basilea, i Vaticinia vennero usati come pamphlet per esprimere l'avversione nei confronti di Felice $\mathrm{V}^{57}$. È poco plausibile ritenere che Miélot sia intervenuto con un pesante commento personale su un personaggio morto nove anni prima della sua traduzione; è molto più probabile che abbia usato un modello che già presentava quest'aggiunta rispetto al testo canonico del vaticinium. A questo punto, appare altrettanto logico supporre che il testo latino copiato sotto la profezia non sia la fonte che Miélot usò per la traduzione ${ }^{58}$.

(52) R. Bossuat, Jean Miélot traducteur de Cicéron cit., p. 105; G. Mombello, Per la fortuna del Boccaccio in Francia. Jean Miélot traduttore di due capitoli della "Genealogia", «Studi sul Boccaccio», 1, 1963, pp. 415-444, in particolare p. 185.

(53) Corsivo mio.

(54) L'inserzione non fa parte della tradizione testuale di Genus nequam. Cfr. M. FLEMING, The Late Medieval Pope Prophecies. The "Genus nequam" Group cit., p. 164.

(55) «Andrac et charboncle, ce sont apostumes petites et pustulles venimeuses et en charboncle est plus grant adustion et le lieu est noir come charbon» in B. DE GoRdon, La Practique de maistre
Bernard de Gordon appellee Fleur de lys en medecine, Lyon, 1495, I, 18. Cfr. Dictionnaire du moyen français 2012, «anthrax».

(56) F. Godefroy, Dictionnaire de l'ancienne langue française et de tous ses dialectes $d u I^{e}$ au XV siècle, Paris, 1880 , vol. 10, p. 822.

(57) H. Millet ha rilevato che i codici prodotti in queste aree ignorano del tutto Felice V. Cfr. H. Millet, "Il libro delle immagini dei Papi". Storia di un testo profetico medievale cit., p. 215.

(58) La parola «corbeaulx» in corrispondenza del latino «cor hominis» potrebbe essere il risultato di un errore paleografico. 
In effetti, immaginando di suddividere il «Papaliste» in due parti, corrispondenti alle antiche serie Ascende calve e Genus nequam, si percepiscono differenze nel rapporto tra le profezie latine e la relativa traduzione in lingua volgare. Nelle profezie derivate dall'Ascende calve, la traduzione francese aderisce perfettamente al testo latino trascritto in calce. Laddove il testo del vaticinium è corrotto, come accade, per esempio, nella seconda profezia [c. $2 \mathrm{v}$ ], la traduzione lo segue in maniera letterale: «Post lunam ascendit mors sitiens sanguinem baptismalem» ${ }^{9} /$ «Aprés la lune monte la mort aiant soif de sang de baptesme». Anche quando le corruzioni riguardano le citazioni bibliche, la traduzione è fedele, come si constata nel caso del versetto II Mac. 6,30, che compone il titolo della terza profezia [c. 3v]: «Duros corporis sustinebis labores $»^{60} /$ «Tu soustendras les durs labeurs du corps». Inoltre, quando i vaticinia aggiungono dei sintagmi rispetto all'Ascende calve, la traduzione li ripropone accuratamente; ecco un esempio tratto dalla tredicesima profezia [c. $13 \mathrm{v}]$ : «Annuncia iustum vel iusticiam ${ }^{61} / \ll$ Annunce le juste ou justice». Infine, si noterà che in questa prima parte dell'opera il testo francese non presenta addizioni rispetto a quello latino ${ }^{62}$.

Al contrario, nelle profezie derivate da Genus nequam, la traduzione francese appare più indipendente dal testo latino che segue: la presenza di numerose lezioni discordanti porta a chiedersi se in questa parte dell'opera Miélot non si sia addirittura basato su due fonti diverse. Qualche esempio potrà servire a chiarire l'ipotesi. Nella quindicesima profezia [c. 15v], il vaticinium riporta «primus filius fere habentis quinque oculos», mentre la traduzione recita «la premiere fin de la beste sauvage ayant V yeulx». L'archetipo Genus nequam ammette le varianti «filius»/«finis», dove «filius» sarebbe la lezione più antica ${ }^{63}$ : Miélot avrebbe dunque potuto tradurre a partire da un modello che presentava la lezione «finis». Un caso analogo si verifica nella diciannovesima profezia [c. 19v]: il vaticinium registra «tertium autem duplicatum primum elementum diversa sunt», mentre la traduzione restituisce «le tiers element double et le premier son $<\mathrm{t}\rangle$ devisez». Ancora una volta l'archetipo ammette le varianti «divisa»/《diversa» ${ }^{64}$, ma la soluzione presente nel testo francese non si accorda a quella del vaticinium sottostante.

Le osservazioni esposte in via preliminare in queste pagine saranno ampliate e approfondite nell'edizione critica del testo, che mi propongo di realizzare in un prossimo futuro; tuttavia, esse consentono già di mostrare l'importanza del manoscritto di Giessen. Da un lato, il codice porta a rivedere la ricezione vernacolare dei Vaticinia de summis pontificibus, dall'altro permette di migliorare la nostra conoscenza della produzione di Miélot. Le carte di questo volume mettono in luce la personalità di un traduttore tutt'altro che passivo, impegnato nel difficile compito di volgarizzare un testo sibillino e denso di corruzioni. Non da ultimo, il ritrovamento del «Papaliste», sconosciuto alla critica per via dell'attribuzione a "Jo. Imelot", induce a chiedersi se

(59) «Post lunam ascendet Mars sciciens sanguinem baptismalem». Cfr. O. SCHWARTZ e R. LERNER, Illuminated Propaganda: The Origins of the "Ascende calve" Pope Prophecies cit., p. 187. Tutti i corsivi nelle citazioni sono miei.

(60) «Duros corporis sustinebit dolores». Cfr. ivi, p. 188.

(61) «Annuncia iustum». Cfr. ivi, p. 191.

(62) La traduzione «edefiera ses portes es troux d'une pierre» [c. 6v] per «in petre foraminibus edificans» costituisce a mio avviso un' eccezione non significativa. «Edificans» è la lezione corrotta di «nidificano» (《in petre foraminibus nidificano»; cfr. Ivi, p. 189); Miélot avrebbe potuto decidere di ag- giungere il complemento oggetto «ses portes» per rendere più chiaro il senso della frase. Allo stesso modo, credo che l'assenza nel vaticinium 13 della frase interna al testo corrispondente alla traduzione «eslieve toy et comme fort et robuste que tu es metz a mort Neron et tu seras asseur» sia semplicemente $\mathrm{da}$ attribuirsi ad un'omissione da parte del copista del manoscritto di Giessen; l'Ascende calve riporta, infatti, la frase «surge, et esto robustus, interfice Neronem, et eris securus». Cfr. ivi, pp. 190-191.

(63) M. Fleming, The Late Medieval Pope Prophecies. The "Genus nequam" Group cit., p. 190.

(64) Ivi, p. 160. 
altri copisti, come nel caso della Vie de saint Foursy, o catalogatori, come per il codice di Giessen, abbiano erroneamente interpretato le lettere che compongono il nome di Miélot: sulla base di questa ipotesi, non è da escludersi che altri rinvenimenti possano un giorno accrescere le nostre conoscenze sulla vita e sull'opera del canonico di Lille. 